\title{
Nanoscale Design of High-Quality Epitaxial Aurivillius Thin Films
}

\author{
Elzbieta Gradauskaite,* Natascha Gray, Marco Campanini, Marta D. Rossell, and Morgan Trassin*
}

Cite This: Chem. Mater. 2021, 33, 9439-9446

Read Online

ABSTRACT: Efforts for the integration of ferroelectric materials in nonvolatile, low energy consuming memories have so far been focused on perovskite oxide materials. Their down-scaling for nanodevices is, however, hindered by finite-size effects, and alternative materials offering more robust polar properties are required. Layered ferroelectrics of the Aurivillius phase have since emerged as promising candidates with robust polarization at subunit-cell thicknesses. Their controlled growth in the epitaxial thin film form has unfortunately remained elusive. Here, we demonstrate the stabilization of the coalescent layer-by-layer growth mode of the $\mathrm{Bi}_{n+1} \mathrm{Fe}_{n-3} \mathrm{Ti}_{3} \mathrm{O}_{3 n+3}$ (BFTO) Aurivillius family homologues. We define the growth conditions for high-quality,

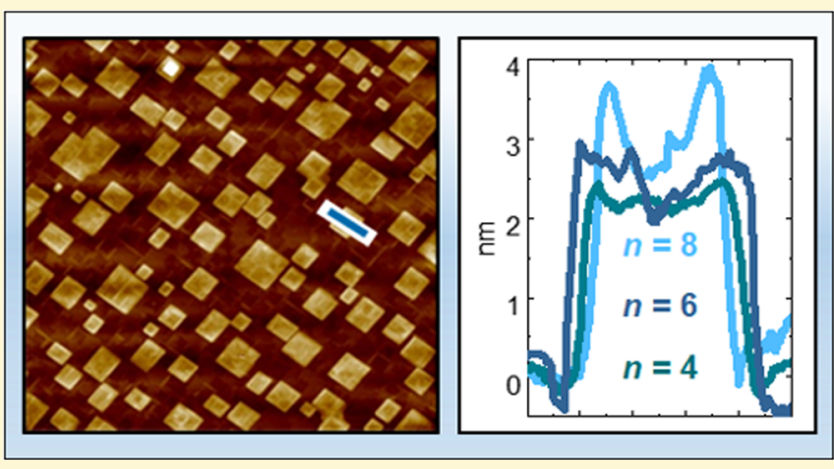
single-crystalline thin films exhibiting ferroelectricity from the first half-unit-cell. We demonstrate the process to be effective for several homologous Aurivillius compositions, which highlights its general applicability. Our work thus provides the systematic framework for the integration of high-quality epitaxial layered ferroelectrics into oxide electronics.

\section{INTRODUCTION}

Ferroelectric oxides, hosting a reversible spontaneous electric polarization, show high potential for emerging low energy consuming, nonvolatile memories. ${ }^{1,2}$ However, their technical implementation in nanoscale devices has not yet been realized owing to finite-size effects. ${ }^{3}$ Efforts are currently underway to find alternatives to classical perovskite ferroelectrics, which lose their net polarization below a ca. 5-unit-cell critical thickness., ${ }^{4,5}$ Therefore, the focus is shifting toward polar systems that remain robust at the nanoscale, such as improper ferroelectrics, ${ }^{6,7} \mathrm{HfO}_{2}$-based ferroelectric thin films, ${ }^{8}$ two-dimensional polar materials, ${ }^{9}$ organic-inorganic hybrid ferroelectrics, $^{10}$ and several classes of layered ferroelectrics. ${ }^{11}$ In particular, layered ferroelectrics of the Aurivillius family ${ }^{12}$ have emerged as promising candidates. They possess a large inplane polarization, ${ }^{13}$ unmatched fatigue resilience, ${ }^{14}$ and the potential for hosting multiferroicity. ${ }^{15}$ Their large and highlyanisotropic unit cell, however, renders their epitaxial integration challenging since impurity phases, ${ }^{16,17}$ crystal twinning, ${ }^{18-20}$ and stacking defects ${ }^{21}$ may form spontaneously during thin-film processing. In light of this, Aurivillius phases have been considered incompatible with nanoscale device architectures up until the recent achievement of high-quality epitaxial films ${ }^{20,22-25}$ with well-defined and tunable physical properties. Furthermore, sub-unit-cell ferroelectricity ${ }^{23,26}$ places Aurivillius compounds at the forefront of ferroelectric oxide research. Nevertheless, systematic studies of epitaxial thin film growth optimization of these compounds with a high degree of control are still lacking.
Here, we establish the optimal epitaxial growth conditions for single-crystalline quality films of Aurivillius phases using a combination of reflection high-energy electron diffraction (RHEED), X-ray diffraction (XRD), and scanning probe microscopy. By stabilizing the coalescent layer-by-layer growth mode during pulsed laser deposition (PLD), we enforce the design of defect-free epitaxial films of Aurivillius phases. By demonstrating this growth mode for a range of $\mathrm{Bi}_{n+1} \mathrm{Fe}_{n-3} \mathrm{Ti}_{3} \mathrm{O}_{3 n+3}$ (BFTO) homologues with even $n$ values ranging from 4 to 8 , we confirm the generality of the process. The resulting films are coherently strained, without any detectable parasitic phases or crystal twinning. The singlecrystalline structure of the films leads to the uniaxial in-plane polarization along one of the substrate axes. For all $n$ studied, we investigate the nanoscale ferroelectric domain configurations and demonstrate polarization switching. The imposed layer-by-layer growth mode of Aurivillius ferroelectrics sets the foundation for the design of high-quality, robust ferroelectric thin films for the next-generation ferroelectric-based oxide electronics.

The BFTO homologous series ${ }^{27-30}$ is one of the most studied families of Aurivillius compounds exhibiting a large in-

Received: October 6, 2021

Revised: November 9, 2021

Published: November 22, 2021 

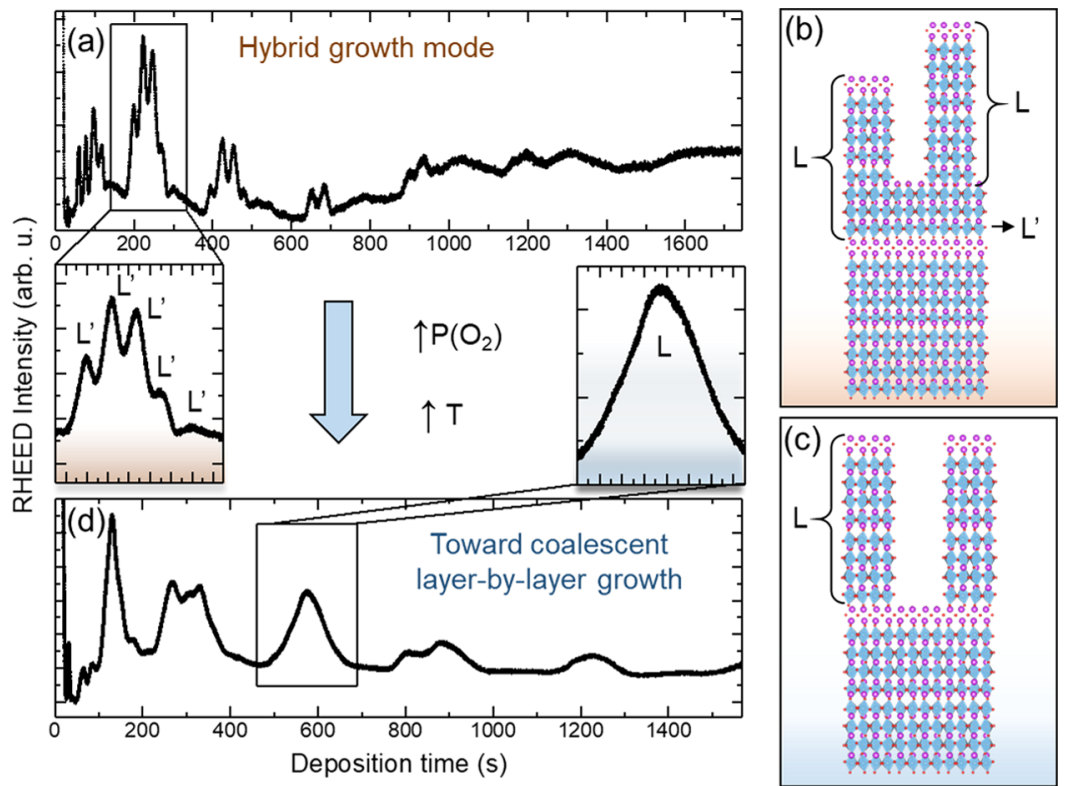

Figure 1. Stabilization of the coalescent layer-by-layer growth mode during the deposition of BFTO. (a) RHEED intensity oscillations during the BFTO $n=8$ growth, showing a hybrid growth mode schematically illustrated in (b). L and L' denote half-unit-cell blocks and perovskite planes, respectively. The coalescent layer-by-layer growth mode, where half-unit-cell height blocks coalesce exclusively, is achieved upon the increase in oxygen pressure and/or temperature with the corresponding representation in (c) and RHEED intensity oscillations shown in (d).

plane polarization, which is uniaxial for even $n .^{31,32}$ The interest in their single-crystalline epitaxial thin films has been fueled by the potential they hold for room-temperature multiferroic device applications. ${ }^{15,33,34}$ This motivates our choice of BFTO as our model system as an alternative to classical perovskite materials. The orthorhombic Aurivillius unit cell comprises alternating layers of a variable number $n$ of perovskite-octahedra planes stacked along the $c$-axis and interleaved between fluorite-like $\left[\mathrm{Bi}_{2} \mathrm{O}_{2}\right]^{2+}$ layers. Thus, these materials are also referred to as natural superlattices. Their highly-anisotropic crystal structure is one of the key obstacles hindering the achievement of single-crystalline Aurivillius thin films. In particular, the orthorhombic unit cell of Aurivillius compounds is not easily matched with standard cubic substrates employed in the routine fabrication of epitaxial films. This in turn leads to crystal twinning. Additionally, the stacking sequence of the layered unit-cell can be lost during the deposition of thin films, leading to the formation of stacking faults and out-of-phase boundaries. ${ }^{21}$

\section{EXPERIMENTAL SECTION}

Thin-Film Growth. BFTO films were grown by PLD at a substrate temperature ranging from 650 to $700{ }^{\circ} \mathrm{C}$. Optimal oxygen partial pressure was found to be $1.5 \times 10^{-2} \mathrm{mbar}$ for BFTO $n=4,5.0$ $\times 10^{-2}$ mbar for $n=6$, and $7.5 \times 10^{-2} \mathrm{mbar}$ for $n=8$. The $\mathrm{KrF}$ excimer laser intensity was set to $0.9 \mathrm{~J} \mathrm{~cm}^{-2}$ for $n=4,6$ and $1.10 \mathrm{~J}$ $\mathrm{cm}^{-2}$ for $n=8$. The thickness of the films was monitored with RHEED and additionally confirmed with X-ray reflectivity (XRR) measurements.

X-ray Diffraction. The crystalline structure of thin films was analyzed by X-ray diffraction and reciprocal space mapping measurements using a four-circle X-ray diffractometer (Panalytical X'Pert MRD).

Scanning Transmission Electron Microscopy. Cross-sectional specimens for transmission electron microscopy analysis were prepared by means of a FEI Helios NanoLab 600i focused-ion beam (FIB) operated at accelerating voltages of 30 and $5 \mathrm{kV}$. The structure of the films was analyzed by high-angle annular dark-field scanning transmission electron microscopy (HAADF-STEM) on a probe-corrected FEI Titan Themis operated at $300 \mathrm{kV}$. The experiments were performed by setting the probe semiconvergence angle to $25 \mathrm{mrad}$ and collecting semiangles for the annular detector of $66-200 \mathrm{mrad}$.

Scanning Probe Microscopy. The piezoresponse force microscopy acquisition was performed using a $5 \mathrm{~V}$ peak-to-peak AC modulation at $70 \mathrm{kHz}$ in contact mode using a NT-MDT NTEGRA Prima scanning probe microscope. Images of in-plane polarized domains were recorded via cantilever torsion mode when measuring with the cantilever perpendicular to the uniaxial polarization axis.

Macroscopic Switching and Ferroelectric Fatigue Testing. Ferroelectric properties of the films were studied using a ferroelectric tester (Radiant Technologies). The remnant polarization during the switching as well as the $P-E$ loops were measured using the positiveup negative-down (PUND) method.

\section{RESULTS AND DISCUSSION}

Coalescent Layer-by-Layer Growth Mode. The homologous BFTO films were grown on lattice-matching orthorhombic $\mathrm{NdGaO}_{3}$ (NGO) (001) substrates by PLD. We use RHEED in situ monitoring to track the structural evolution of our thin films during the growth. The layer-by-layer growth mode is usually desired for the deposition of epitaxial thin films. It enables real-time thickness tracking, for which each peak of the integrated RHEED intensity corresponds to a complete layer of the material deposited. In standard perovskite oxides, this is equal to the deposition of a layer with the height of a cubic perovskite unit cell. ${ }^{35}$ In layered compounds with complex unit cells, however, this no longer holds true, and one oscillation may correspond to a fraction of the unit cell. ${ }^{23,35-37}$ Therefore, we use ex situ X-ray reflectivity (XRR) and atomic force microscopy (AFM) in combination with RHEED monitoring to shed light on the growth mechanism with sub-unit-cell accuracy.

To investigate the growth mechanism, we monitor the RHEED intensity during the BFTO $n=8$ growth (Figure 1 ). In nonoptimized growth conditions (Figure 1a), the variations 
(a)

(b)
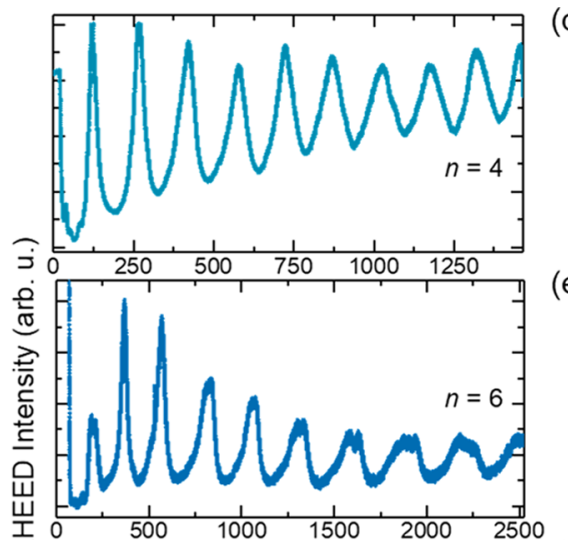

(c)

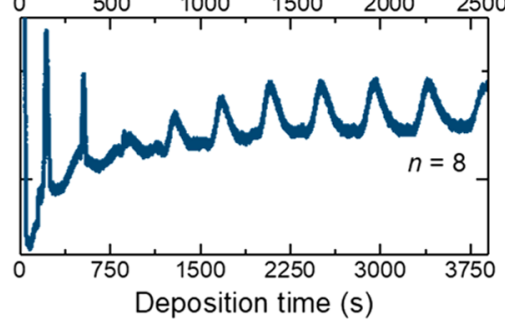

(d)

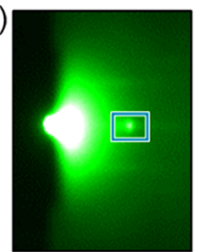

(e)

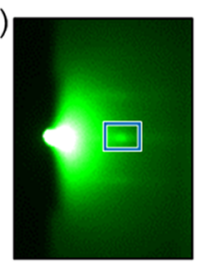

(f)

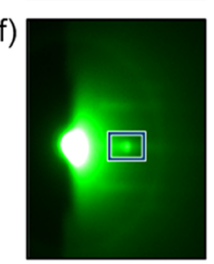

(g)

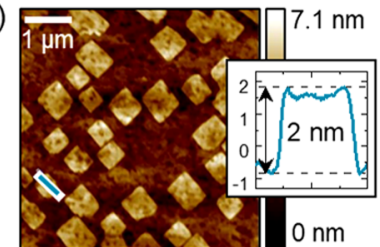

(h)

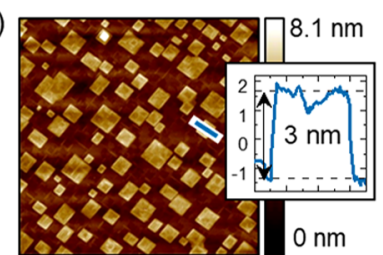

(i)

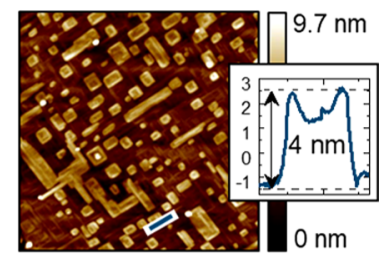

Figure 2. Coalescent layer-by-layer growth mode of BFTO homologues. (a-c) RHEED intensity oscillations during the layer-by-layer growth of $n$ $=4,6,8$ members of BFTO homologous series, respectively. $(\mathrm{d}-\mathrm{f})$ Corresponding RHEED patterns characteristic of two-dimensional crystal surfaces recorded for each growth, respectively. The intensity variations were tracked for the reflections that are indicated by the rectangular frames. $(\mathrm{g}-\mathrm{i})$ Corresponding AFM topography scans of the three BFTO films showing cuboids on atomically flat surfaces. The cross-sectional topography profiles (in the insets) show the height of these features to be $\sim \mathcal{c} / 2$.

of RHEED intensity during the film deposition consist of a mixture of oscillating contributions. We identify a set of lowfrequency oscillations superimposed with higher frequency oscillations of lower amplitude. Ex situ XRR measurements were performed after interrupting the growth at different stages of RHEED monitoring to find the relation between oscillations in intensity and the resulting thickness of the films. We found that the measured film thicknesses cannot be rationalized by the count of high amplitude oscillations only, previously linked to the layer-by-layer growth via coalescence of half-unit-cell height blocks. ${ }^{23}$ Here, additional layers of perovskite-type planes have to be considered. This suggests the presence of different $n$ intergrowths in the films and allows us to ascribe the higher period oscillations to an additional, spontaneous layering of perovskite-type planes. All of these observations are consistent with a hybrid growth mode, during which the film grows via the simultaneous coalescence of half-unit-cell blocks (L) and perovskite planes (L'), as sketched in Figure $1 \mathrm{~b}$. In this growth mode, the number of perovskite layers in the structure is not governed by the target stoichiometry and varies from one Aurivillius unit cell to the other. This is evidenced by the inconsistent count of low amplitude peaks (perovskite-type layers) that appear simultaneously with high amplitude oscillations (half-unit-cell blocks). All this reveals the complexity of the layer ordering in the growth direction and suggests an absence of a coherent, deterministic growth mode in the nonoptimized conditions.

We propose that stabilizing the layer-by-layer growth mode, where blocks of exclusively half-unit-cell height coalesce uniformly to form full layer coverage, is key to the achievement of defect-free Aurivillius films, as represented in Figure 1c. The layer-by-layer or Frank-van der Merwe ${ }^{38}$ growth mode can be achieved for perovskite oxides ${ }^{39}$ in a specific range of deposition temperatures and deposition rates, ${ }^{40}$ where the growth dynamics favors full coverage of the deposited species before the growth of the next layer starts. Hence, we systematically varied the growth pressure and temperature to establish the growth conditions where such layer-by-layer growth occurs. Figure 1d shows the RHEED signature of transition toward coalescent layer-by-layer growth mode of the films grown at $700{ }^{\circ} \mathrm{C}$ at $7.5 \times 10^{-2}$ mbar oxygen partial pressure. Ex situ XRR measurements reveal that each RHEED oscillation now corresponds to exactly one-half of the Aurivillius unit cell height. Hence, a ferroelectric unit cell of pure Aurivillius phase is deposited every two RHEED oscillations. The RHEED pattern evolution and its relationship to the structural development during the growth are shown in Figure S1. Here, half-unit-cell-high blocks coalesce to form full layers. This feature is likely to be related to the layered and charged structure of Aurivillius compounds: positively charged $\left[\mathrm{Bi}_{2} \mathrm{O}_{2}\right]^{2+}$ layers alternating with negatively charged $\left[\mathrm{A}_{n-1} \mathrm{~B}_{n} \mathrm{O}_{3 n+1}\right]^{2-}$ perovskite planes. This growth mode was consistently established in numerous samples prepared with substrate temperatures ranging from 650 to $720^{\circ} \mathrm{C}$ and oxygen pressure varied from $1.5 \times 10^{-2}$ to $7.5 \times 10^{-1} \mathrm{mbar}$.

In Figure $2 a-c$, we show the generality of our imposed coalescent layer-by-layer growth mode for all three BFTO homologues $n=4,6$, and 8. RHEED oscillations steadily persist for all BFTO compositions, enabling the highly controlled fabrication of Aurivillius films as thick as tens of nanometers. The corresponding RHEED patterns are shown in Figure $2 \mathrm{~d}-\mathrm{f}$. Note that higher oxygen partial pressure and higher laser fluence were beneficial upon the increase in $n$ value (see Experimental Section for details). Topography scans of the three films (Figure $2 \mathrm{~g}-\mathrm{i}$ ) feature sharply pronounced cuboids protruding the atomically flat surface. From the crosssectional profiles (Figure $2 \mathrm{~g}-\mathrm{i}$, right), the height of the cuboids was estimated to be about 2,3 , and $4 \mathrm{~nm}$ for $n=4,6$, 
(a)

Out-of-phase boundaries

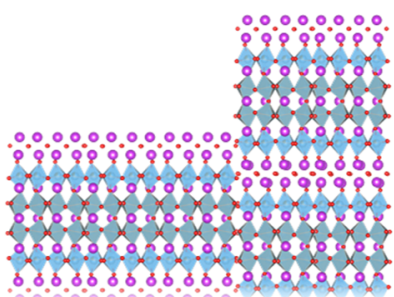

(b)
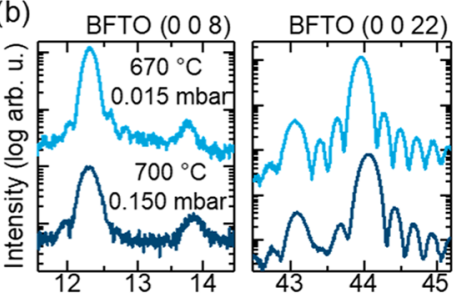

(d)

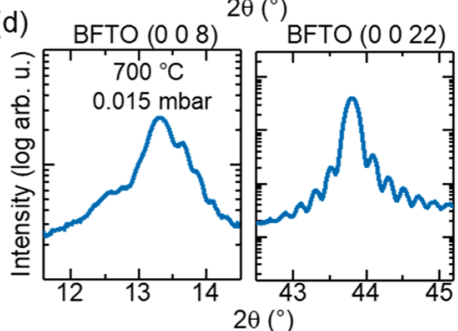

(c)

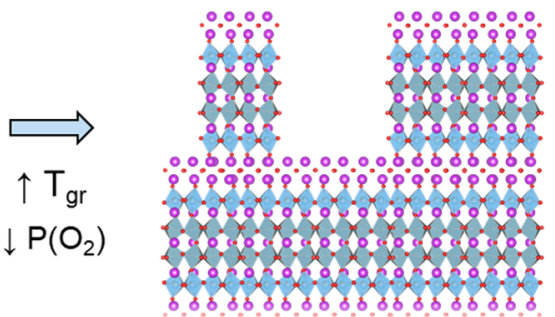

(e)

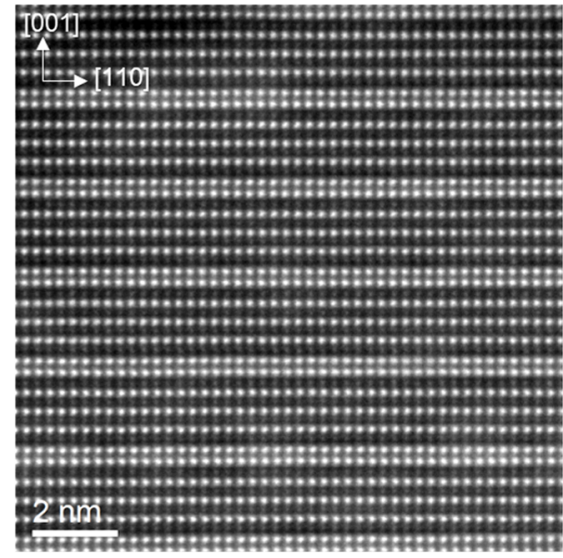

Figure 3. OPB control during the growth of BFTO $n=4$ films. (a) Sketch of BFTO film exhibiting OPBs. A signature of such defects appears in $\theta-$ $2 \theta$ XRD patterns as the $\left(\begin{array}{lll}0 & 0 & l\end{array}\right)$ peak splitting. Selected $\left(\begin{array}{lll}0 & 0 & 8\end{array}\right)$ and $\left(\begin{array}{lll}0 & 0 & 22\end{array}\right)$ reflexes are shown in (b). (c) Sketch of a defect-free film achieved upon the increase in deposition temperature and decrease in oxygen pressure, which displays no signatures of peak splitting in the ( 008$)$ and ( 0022$)$ reflexes in $\theta-2 \theta$ XRD pattern, shown in (d). (e) High-resolution HAADF-STEM image of a BFTO $n=4$ film grown on NGO (001).
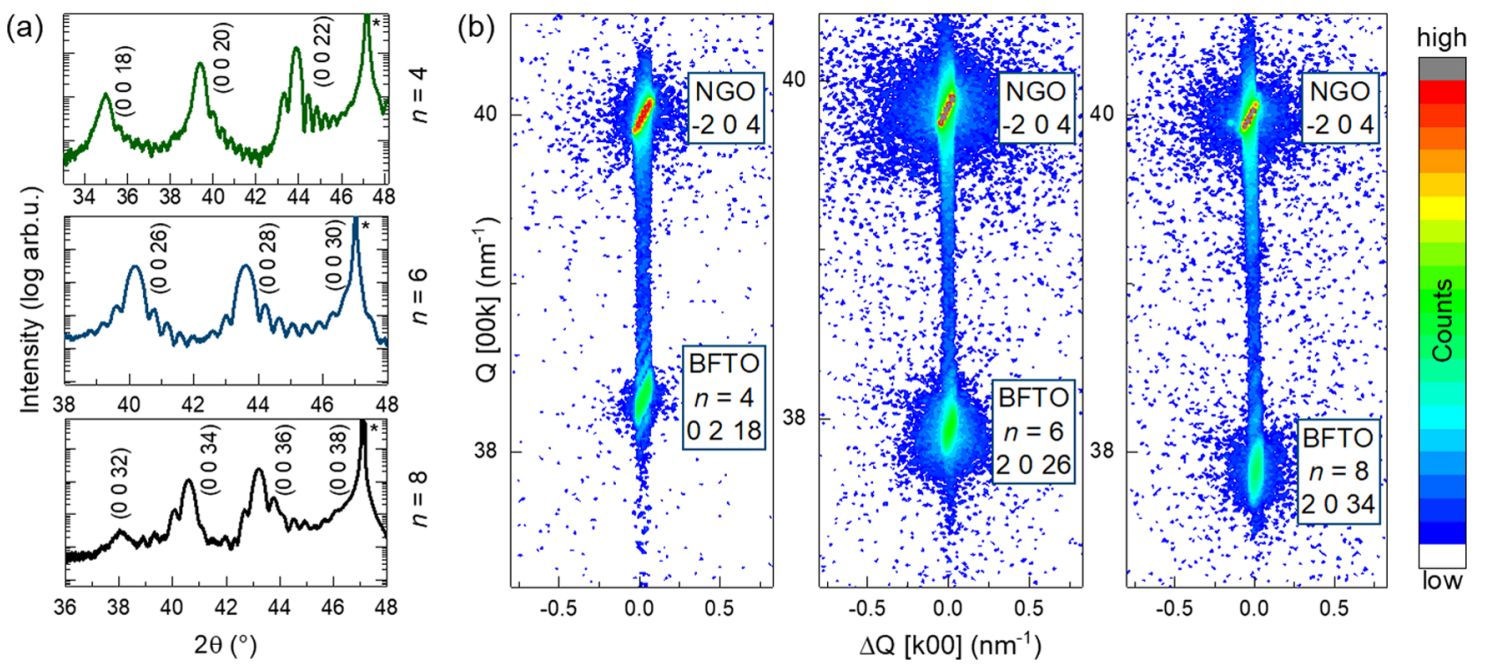

Figure 4. Structural characterization of homologous BFTO thin films. (a) $\theta-2 \theta$ XRD patterns around the NGO (004) substrate peak (*) indicating 001-oriented epitaxial orthorhombic BFTO thin films of $n=4,6$, and 8 compositions. (b) Reciprocal space maps around the NGO $(-204)$ substrate peak reveal a coherent epitaxial strain in all three BFTO homologues.

and 8 , respectively. These values match the half $c$-axis lattice parameter of each unit cell of the three homologues, i.e., $2.1{ }^{41}$ $2.9,{ }^{27}$ and $3.9 \mathrm{~nm},{ }^{42}$ respectively. This, in combination with in situ RHEED and XRD analysis indicating the preservation of the Aurivillius phase during the growth, allows us to conclude that the topographic features correspond to half-unit-cell blocks. They appear as a result of the incomplete layer coverage $^{39}$ and hence are the signatures of the coalescent layerby-layer growth mode in our $n=4,6$, and 8 BFTO thin films.
In conclusion, RHEED monitoring during the layer-by-layer growth mode enables the sub-unit-cell control in our epitaxial Aurivillius layers-a cornerstone for their integration into nanoscale oxide devices.

Control over Out-of-Phase Boundary (OPB) Formation. In situ RHEED diagnostic tool renders the controlled deposition of complex layered oxide materials possible. It remains, however, insensitive to the stacking faults in the single-phase Aurivillius thin films, such as out-of-phase 

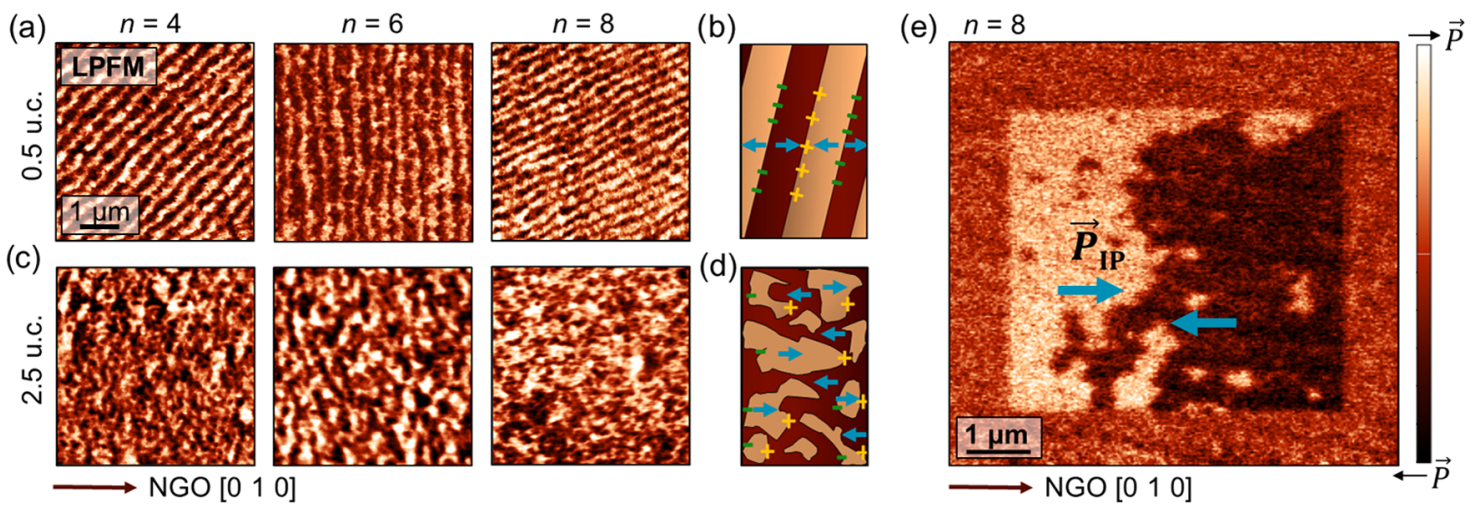

Figure 5. Ferroelectric domain configuration in homologous BFTO films in two different thickness regimes. (a) In the ultrathin regime of 0.5 uc thickness, all three BFTO films exhibit in-plane-polarized stripe domains. The stripe-domain configuration, in conjunction with uniaxial in-plane polarization (along the NGO [0 10$]$-direction), results in alternating tail-to-tail and head-to-head nominally charged domain walls, shown schematically in (b). (c) In the thicker regime of $2.5 \mathrm{uc}$ and above, all BFTO films contain small, randomly-distributed in-plane domains, as represented in (d). (e) Local in-plane domain switching in BFTO $n=8$ film using trailing in-plane field resulting from the DC voltage (5 V) applied to the tip. The increased size of the domains and resulting changes in the length and orientation of the charged domain wall are visualized with LPFM.

boundaries $(\mathrm{OPBs}) .^{21} \mathrm{OPBs}$ are translation boundary defects routinely imaged with STEM $^{21,43}$ as an out-of-phase unit cell shift in the vertical direction by a fraction of unit cell (uc). Such structural defects have been linked to inhomogeneous strain fields ${ }^{44}$ and, in some instances, even to suppressed polarization. ${ }^{45,46}$ Recent experiments further highlighted their impact on the topology of polar states in ferroelectric thin films. ${ }^{47}$ Hence, their occurrence should be controlled to perfect the ferroelectric performance.

We therefore investigate OPBs, which can be conveniently traced using XRD. The out-of-phase alignment of crystallographic domains within a diffracting volume results in the splitting of selected $(00 \mathrm{l})$ peaks. ${ }^{21,48}$ The density of OPBs in a material $^{21,48}$ has been reported to be directly correlated with the degree of peak splitting. BFTO $n=4$ films, grown in the layer-by-layer mode, exhibit signatures of OPBs (Figure 3a) in the $\left(\begin{array}{lll}0 & 0 & 8\end{array}\right)$ and $\left(\begin{array}{lll}0 & 0 & 22\end{array}\right)$ reflexes (Figure $\left.3 b\right)$. By tuning the deposition conditions while maintaining the layer-by-layer mode, we found that the substrate temperature and partial oxygen pressure have the strongest influence on $\mathrm{OPB}$ formation. In particular, high deposition temperatures $(\geq 700$ $\left.{ }^{\circ} \mathrm{C}\right)$ and lower oxygen pressure $\left(\leq 1.5 \times 10^{-2} \mathrm{mbar}\right)$ minimize the nucleation of OPBs and result in defect-free films (Figure $3 c$ ). Accordingly, the peak splitting disappears in the aforementioned reflexes in Figure $3 \mathrm{~d}$. Increased growth temperature and lowered oxygen partial pressure both result in increased energy of the deposited species. This boosts their ability to rearrange post-deposition to achieve higher film crystallinity. According to our study, the post-deposition rearrangement appears to be the most significant factor in the OPB formation in thin Aurivillius films. Additional local high-angle annular dark-field scanning transmission electron microscopy (HAADF-STEM) confirms the high quality of the film and allows to directly visualize the expected $n=4$ superlattice layering, as shown in Figure 3e.

Structural Characterization of BFTO Films. We show the structural characterization of all three homologous BFTO films prepared in the optimal growth conditions in Figure 4. $\theta-2 \theta$ XRD scans recorded close to the NGO $\left(\begin{array}{lll}0 & 0 & 4\end{array}\right)$ substrate peak reveal $(00 l)$ reflections of the three samples consistent with the $c$-orientation of the films (Figure $4 a$, for full scan ranges, see Figure S2). All XRD reflections are assigned to
BFTO films, suggesting the absence of parasitic phases in the sensitivity limits of our XRD experiments. High crystalline quality of the three films is further confirmed by Laue thickness fringes. Reciprocal space mapping around NGO (-204) (Figure 4b) provides direct evidence that all three BFTO films are coherently strained to the substrate with increasing $c$ parameter reflected in the changing $\mathrm{Q}\left[\begin{array}{lll}0 & 0 & k\end{array}\right]$ position (for unit cell parameters and epitaxial strain values see Supporting Information, Note 3). No strain relaxation is observed with increasing thickness, thanks to the excellent lattice-matching between BFTO homologues and NGO (001). ${ }^{23,43}$ The epitaxial stabilization of the expected orthorhombic phases further grants our confidence in the phase purity. This demonstrates our ability to design fully strained, epitaxial Aurivillius thin films, free of crystal twinning and detectable stacking faults.

Ferroelectric Properties of Ultrathin BFTO Films. Next, we characterize the functional properties of our growthoptimized BFTO layers. Here, for all our $n$ values that are even, we expect room-temperature uniaxial in-plane ferroelectricity $^{23,31,32}$ from the first half-unit-cell. ${ }^{23}$ Vectorial piezoresponse force microscopy (PFM) is the perfect tool for the investigation of anisotropic in-plane ferroelectric response in thin films. It unambiguously disentangles in-plane piezoresponse from other contributions and assigns the precise polarization orientation within each domain with respect to the Aurivillius phase crystalline orientations. ${ }^{17,23}$ Here, the uniaxial in-plane polarization lies along the longer in-plane lattice parameter of the Aurivillius unit cells, corresponding to NGO $\left[\begin{array}{lll}0 & 1 & 0\end{array}\right]$. It is best captured by monitoring the cantilever torsion. This torsion has a maximum response when the cantilever lies perpendicular to the polar axis of the film. Therefore, we scan with the cantilever oriented perpendicular to the NGO $\left[\begin{array}{lll}0 & 1 & 0\end{array}\right]$-direction. In this measurement geometry, left and right polarization directions correspond to the dark and bright contrast in lateral PFM (LPFM) channel, respectively. Note that the vertical PFM (VPFM) channel is measured simultaneously. In the present case, however, as expected, no signal was recorded in this channel (see Figure S3).

RHEED monitoring enables the deposition of exactly one half-unit-cell-thick BFTO films of all three $n$ homologues. All 
ultrathin layers exhibit polar domain patterns and hence, unambiguous ferroelectric response (Figure 5a). Note that reversible switching of 0.5 uc-thick Aurivillius layers was demonstrated previously. ${ }^{26}$ We confirm the uniaxial in-plane polarization along the NGO [0 10 l-direction, which corroborates the absence of crystal twinning in the films. All three films exhibit stripe in-plane-polarized domains, which result in an array of alternating nominally charged head-to-head and tail-to-tail domain walls (sketched in Figure $5 \mathrm{~b}$ ), as previously characterized at the nanoscale using STEM. ${ }^{47}$ The precise control that we have over the thickness of our films allows us to probe interlayer coupling for BFTO homologues of different $n$. Here, independent of $n$, we see a deviation from ordered stripe domains with charged domain walls to a random distribution of small in-plane domains for increasing thickness starting from 2.5 uc (Figure 5c,d). Thus, we can accurately and universally control the domain configuration through the thickness of the films in this Aurivillius family. As different $n$ values are associated with dissimilar remnant polarization and ferroelectric transition temperature values, ${ }^{28}$ the ability to grow layers of any Aurivillius homologue offers a way to engineer these parameters in functional oxide electronics.

Lastly, we demonstrate that domain configuration in these films can be modified at will. The in-plane trailing field originating from the scanning probe tip can move the domain walls ${ }^{17,23}$ in the three BFTO films despite the absence of metallic top and bottom electrodes. Figure 5e shows a poled area in the $n=8$ BFTO film with enlarged areas of both domain types. Identical poling procedures were accomplished in $n=4$ and $n=6$ films. Thus, the charged domain wall configuration, i.e., length, orientation, and geometry, can be manipulated on demand, which is of interest for domain wallbased nano-circuitry. ${ }^{49-51}$ In addition, the macroscopic switching of the in-plane polarization measured in a representative $n=4$ BFTO film of 8.5 uc (see Figure S4) shows a ferroelectric hysteresis with a saturation polarization of $10.5 \mu \mathrm{C} \mathrm{cm}^{-2}$. Fatigue measurements further confirm the robust switching properties of the film with little degradation in switchable polarization even after $10^{9}$ switching cycles (see Figure S4).

\section{CONCLUSIONS}

In summary, we demonstrate the growth and characterization of ultrathin high-quality single-crystalline films of the BFTO Aurivillius family. The epitaxial deposition of films of the three homologues $n=4,6$, and 8 is achieved via the coalescent layerby-layer growth mode. RHEED in situ monitoring enables the design of films that are free of detectable parasitic phases, crystalline twinning, and stacking faults. For ultrathin films of all $n$, we verify uniaxial in-plane ferroelectricity with tunable configurations of charged domain walls and thickness-dependent domain architecture. Both structural and polar properties can be engineered at the nanoscale throughout the homologous series of the BFTO family. With this, we provide a versatile toolkit for the integration of layered ferroelectrics into oxide electronics.

\section{ASSOCIATED CONTENT}

\section{(s) Supporting Information}

The Supporting Information is available free of charge at https://pubs.acs.org/doi/10.1021/acs.chemmater.1c03466.
RHEED intensity and pattern variations during the coalescent layer-by-layer growth, full-range $\theta-2 \theta$ XRD patterns of $n=4,6$, and 8 BFTO thin films, epitaxial relationship between BFTO films and NGO (001) substrate, PFM measurement geometry, and macroscopic switching properties (PDF)

\section{AUTHOR INFORMATION}

\section{Corresponding Authors}

Elzbieta Gradauskaite - Department of Materials, ETH Zurich, 8093 Zurich, Switzerland; ㅇorcid.org/0000-00019607-1855; Email: elzbieta.gradauskaite@mat.ethz.ch

Morgan Trassin - Department of Materials, ETH Zurich, 8093 Zurich, Switzerland; (ㅇ) orcid.org/0000-0002-02400257; Email: morgan.trassin@mat.ethz.ch

\section{Authors}

Natascha Gray - Department of Materials, ETH Zurich, 8093 Zurich, Switzerland

Marco Campanini - Electron Microscopy Center, Empa, 8600 Dübendorf, Switzerland; 다이.org/0000-0003-43124794

Marta D. Rossell - Electron Microscopy Center, Empa, 8600 Dübendorf, Switzerland; 다이.org/0000-0001-86108853

Complete contact information is available at:

https://pubs.acs.org/10.1021/acs.chemmater.1c03466

\section{Author Contributions}

All authors discussed the results and contributed to the completion of the manuscript. E.G. performed the thin film growth, structural characterization, ferroelectric characterization, and PFM analysis together with N.G. M.C. and M.D.R. carried out the STEM analysis. M.T. designed the experiment with E.G. and supervised the work.

\section{Notes}

The authors declare no competing financial interest.

\section{ACKNOWLEDGMENTS}

E.G. and M.T. acknowledge the financial support by the Swiss National Science Foundation under Project No. 200021188414. M.C. and M.D.R. acknowledge financial support by the Swiss National Science Foundation under Project No. 200021-175926. The authors acknowledge B. Biswas and C. W. Schneider for the design of interdigitated electrodes used for the macroscopic ferroelectric characterization. The authors thank Mads C. Weber and Thomas Weber for fruitful discussions and Manfred Fiebig for financial support.

\section{REFERENCES}

(1) Scott, J. F. Ferroelectric Memories; Springer-Verlag: New York, 2000; pp 1-243.

(2) Gradauskaite, E.; Meisenheimer, P.; Müller, M.; Heron, J.; Trassin, M. Multiferroic heterostructures for spintronics. Phys. Sci. Rev. 2021, 6, No. 20190072.

(3) Fong, D. D.; Stephenson, G. B.; Streiffer, S. K.; Eastman, J. A.; Aucielo, O.; Fuoss, P. H.; Thompson, C. Ferroelectricity in ultrathin perovskite films. Science 2004, 304, 1650-1653.

(4) Junquera, J.; Ghosez, P. Critical thickness for ferroelectricity in perovskite ultrathin films. Nature 2003, 422, 506-509.

(5) De Luca, G.; Strkalj, N.; Manz, S.; Bouillet, C.; Fiebig, M.; Trassin, M. Nanoscale design of polarization in ultrathin ferroelectric heterostructures. Nat. Commun. 2017, 8, No. 1419. 
(6) Li, M.; Tan, H.; Duan, W. Hexagonal rare-earth manganites and ferrites: A review of improper ferroelectricity, magnetoelectric coupling, and unusual domain walls. Phys. Chem. Chem. Phys. 2020, 22, 14415-14432.

(7) Bousquet, E.; Dawber, M.; Stucki, N.; Lichtensteiger, C.; Hermet, P.; Gariglio, S.; Triscone, J. M.; Ghosez, P. Improper ferroelectricity in perovskite oxide artificial superlattices. Nature 2008, 452, 732-736.

(8) Park, M. H.; Lee, Y. H.; Mikolajick, T.; Schroeder, U.; Hwang, C. S. Review and perspective on ferroelectric $\mathrm{HfO}_{2}$-based thin films for memory applications. MRS Commun. 2018, 8, 795-808.

(9) Guan, Z.; Hu, H.; Shen, X.; Xiang, P.; Zhong, N.; Chu, J.; Duan, C. Recent Progress in Two-Dimensional Ferroelectric Materials. Adv. Electron. Mater. 2020, 6, No. 1900818.

(10) Xu, W. J.; Kopyl, S.; Kholkin, A.; Rocha, J. Hybrid organicinorganic perovskites: Polar properties and applications. Coord. Chem. Rev. 2019, 387, 398-414.

(11) Benedek, N. A.; Rondinelli, J. M.; Djani, H.; Ghosez, P.; Lightfoot, P. Understanding ferroelectricity in layered perovskites: New ideas and insights from theory and experiments. Dalton Trans. 2015, 44, 10543-10558.

(12) Aurivillius, B. Mixed Bismuth Oxides with Layer Lattices. 1. The Structure Type of $\mathrm{CaNb}_{2} \mathrm{Bi}_{2} \mathrm{O}_{9}$. Ark. Kemi 1949, 1, 463-480.

(13) Newnham, R. E.; Wolfe, R. W.; Dorrian, J. F. Structural basis of ferroelectricity in the bismuth titanate family. Mater. Res. Bull. 1971, 6, $1029-1039$

(14) de Araujo, C. A.-P.; Cuchiaro, J. D.; McMillian, L. D.; Scott, M. C.; Scott, J. F. Fatigue-free ferroelectric capacitors with platinum electrodes. Nature 1995, 374, 627-629.

(15) Birenbaum, A. Y.; Ederer, C. Potentially multiferroic Aurivillius phase $\mathrm{Bi}_{5} \mathrm{FeTi}_{3} \mathrm{O}_{15}$ : Cation site preference, electric polarization, and magnetic coupling from first principles. Phys. Rev. B 2014, 90, No. 214109.

(16) Faraz, A.; Maity, T.; Schmidt, M.; Deepak, N.; Roy, S.; Pemble, M. E.; Whatmore, R. W.; Keeney, L. Direct visualization of magneticfield-induced magnetoelectric switching in multiferroic aurivillius phase thin films. J. Am. Ceram. Soc. 2017, 100, 975-987.

(17) Keeney, L.; Saghi, Z.; O'Sullivan, M.; Alaria, J.; Schmidt, M.; Colfer, L. Persistence of Ferroelectricity Close to Unit-Cell Thickness in Structurally Disordered Aurivillius Phases. Chem. Mater. 2020, 32, 10511-10523.

(18) Lettieri, J.; Zurbuchen, M. A.; Jia, Y.; Schlom, D. G.; Streiffer, S. K.; Hawley, M. E. Epitaxial growth of $\mathrm{SrBi}_{2} \mathrm{Nb}_{2} \mathrm{O}_{9}$ on (110) $\mathrm{SrTiO}_{3}$ and the establishment of a lower bound on the spontaneous polarization of $\mathrm{SrBi}_{2} \mathrm{Nb}_{2} \mathrm{O}$. Appl. Phys. Lett. 2000, 77, 3090-3092.

(19) Lee, H. N.; Hesse, D.; Zakharov, N.; Lee, S. K.; Gösele, U. Growth of uniformly a -axis-oriented ferroelectric lanthanumsubstituted bismuth titanate films on silicon substrates. J. Appl. Phys. 2003, 94, 2148.

(20) Wang, C.; Ke, X.; Wang, J.; Liang, R.; Luo, Z.; Tian, Y.; Yi, D.; Zhang, Q.; Wang, J.; Han, X. F.; Van Tendeloo, G.; Chen, L. Q.; Nan, C. W.; Ramesh, R.; Zhang, J. Ferroelastic switching in a layeredperovskite thin film. Nat. Commun. 2016, 7, No. 10636.

(21) Zurbuchen, M. A.; Tian, W.; Pan, X. Q.; Fong, D.; Streiffer, S. K.; Hawley, M. E.; Lettieri, J.; Jia, Y.; Asayama, G.; Fulk, S. J.; Comstock, D. J.; Knapp, S.; Carim, A. H.; Schlom, D. G. Morphology, structure, and nucleation of out-of-phase boundaries (OPBs) in epitaxial films of layered oxides. J. Mater. Res. 2007, 22, 1439-1471.

(22) Cao, X.; Liu, Z.; Dedon, L. R.; Bell, A. J.; Esat, F.; Wang, Y.; Yu, P.; Wang, C.; Jin, P. Epitaxial $\mathrm{Bi}_{9} \mathrm{Ti}_{3} \mathrm{Fe}_{5} \mathrm{O}_{27}$ thin films: A new type of layer-structure room-temperature multiferroic. J. Mater. Chem. C 2017, 5, 7720-7725.

(23) Gradauskaite, E.; Campanini, M.; Biswas, B.; Schneider, C. W.; Fiebig, M.; Rossell, M. D.; Trassin, M. Robust In-Plane Ferroelectricity in Ultrathin Epitaxial Aurivillius Films. Adv. Mater. Interfaces 2020, 7, No. 2000202.

(24) Jeong, J.; Mun, J.; Das, S.; Kim, J.; Kim, J. R.; Peng, W.; Kim, M.; Noh, T. W. Growth and Atomically Resolved Polarization
Mapping of Ferroelectric $\mathrm{Bi}_{2} \mathrm{WO}_{6}$ Thin Films. ACS Appl. Electron. Mater. 2021, 3, 1023-1030.

(25) Das, S.; Ohkubo, T.; Kasai, S.; Kozuka, Y. Deterministic influence of substrate-induced oxygen vacancy diffusion on $\mathrm{Bi}_{2} \mathrm{WO}_{6}$ thin film growth. Cryst. Growth Des. 2021, 21, 625-630.

(26) Keeney, L.; Smith, R. J.; Palizdar, M.; Schmidt, M.; Bell, A. J.; Coleman, J. N.; Whatmore, R. W. Ferroelectric Behavior in Exfoliated 2D Aurivillius Oxide Flakes of Sub-Unit Cell Thickness. Adv. Electron. Mater. 2020, 6, No. 1901264.

(27) Krzhizhanovskaya, M.; Filatov, S.; Gusarov, V.; Paufler, P.; Bubnova, R.; Morozov, M.; Meyer, D. C. Aurivillius phases in the $\mathrm{Bi}_{4} \mathrm{Ti}_{3} \mathrm{O}_{12} / \mathrm{BiFeO}_{3}$ system: Thermal behaviour and crystal structure. Z. Anorg. Allg. Chem. 2005, 631, 1603-1608.

(28) Lomanova, N. A.; Morozov, M. I.; Ugolkov, V. L.; Gusarov, V. V. Properties of Aurivillius phases in the $\mathrm{Bi}_{4} \mathrm{Ti}_{3} \mathrm{O}_{12}-\mathrm{BiFeO}_{3}$ system. Inorg. Mater. 2006, 42, 189-195.

(29) Song, D.; Yang, J.; Yang, B.; Chen, L.; Wang, F.; Zhu, X. Evolution of structure and ferroelectricity in Aurivillius $\mathrm{Bi}_{4} \mathrm{Bi}_{\mathrm{n}-3} \mathrm{Fe}_{\mathrm{n}-3} \mathrm{Ti}_{3} \mathrm{O}_{3 \mathrm{n}+3}$ thin films. J. Mater. Chem. C 2018, 6, 8618.

(30) Sun, S.; Yin, X. Progress and perspectives on aurivillius-type layered ferroe-lectric oxides in binary $\mathrm{Bi}_{4} \mathrm{Ti}_{3} \mathrm{O}_{12}-\mathrm{BiFeO}_{3}$ system for multifunctional applications. Crystals 2021, 11, No. 23.

(31) Funakubo, H. Degradation-free dielectric property using bismuth layer-structured dielectrics having natural superlattice structure. J. Ceram. Soc. Jpn. 2008, 116, 1249-1254.

(32) Zhang, P. F.; Deepak, N.; Keeney, L.; Pemble, M. E.; Whatmore, R. W. The structural and piezoresponse properties of caxis-oriented Aurivillius phase $\mathrm{Bi}_{5} \mathrm{Ti}_{3} \mathrm{FeO}_{15}$ thin films deposited by atomic vapor deposition. Appl. Phys. Lett. 2012, 101, No. 112903.

(33) Keeney, L.; Maity, T.; Schmidt, M.; Amann, A.; Deepak, N.; Petkov, N.; Roy, S.; Pemble, M. E.; Whatmore, R. W. Magnetic FieldInduced Ferroelectric Switching in Multiferroic Aurivillius Phase Thin Films at Room Temperature. J. Am. Ceram. Soc. 2013, 96, 23392357.

(34) Trassin, M. Low energy consumption spintronics using multiferroic heterostructures. J. Phys.: Condens. Matter 2016, 28, No. 033001.

(35) Sarott, M. F.; Gradauskaite, E.; Nordlander, J.; Strkalj, N.; Trassin, M. In situ monitoring of epitaxial ferroelectric thin-film growth. J. Phys.: Condens. Matter 2021, 33, No. 293001.

(36) Homkar, S.; Preziosi, D.; Devaux, X.; Bouillet, C.; Nordlander, J.; Trassin, M.; Roulland, F.; Lefêvre, C.; Versini, G.; Barre, S.; Leuvrey, C.; Lenertz, M.; Fiebig, M.; Pourroy, G.; Viart, N. Ultrathin regime growth of atomically flat multiferroic gallium ferrite films with perpendicular magnetic anisotropy. Phys. Rev. Mater. 2019, 3, No. 124416.

(37) Nordlander, J.; Rossell, M. D.; Campanini, M.; Fiebig, M.; Trassin, M. Epitaxial integration of improper ferroelectric hexagonal $\mathrm{YMnO}_{3}$ thin films in heterostructures. Phys. Rev. Mater. 2020, 4, No. 124403.

(38) Frank, F. C.; Van Der Merwe, J. H. One-dimensional dislocations. I. Static theory. Proc. R. Soc. A 1949, 198, 205-216.

(39) Koster, G.; Huijben, M.; Janssen, A.; Rijnders, G. Growth Studies of Heteroepitaxial Oxide Thin Films Using Reflection HighEnergy Electron Diffraction (RHEED); Elsevier Ltd., 2015; pp 3-29.

(40) Venables, J. A.; Spiller, G. D. T.; Hanbücken, M. Nucleation and growth of thin films. Rep. Prog. Phys. 1984, 47, 399-459.

(41) Hervoches, C. H.; Snedden, A.; Riggs, R.; Kilcoyne, S. H.; Manuel, P.; Lightfoot, P. Structural behavior of the four-layer aurivillius-phase ferroelectrics $\mathrm{SrBi}_{4} \mathrm{Ti}_{4} \mathrm{O}_{15}$ and $\mathrm{Bi}_{5} \mathrm{Ti}_{3} \mathrm{FeO}_{15}$. J. Solid State Chem. 2002, 164, 280-291.

(42) Patri, S. K.; Choudhary, R. N.; Samantaray, B. K. Studies of structural, dielectric and impedance properties of $\mathrm{Bi}_{9} \mathrm{Fe}_{5} \mathrm{Ti}_{3} \mathrm{O}_{27}$ ceramics. J. Electroceram. 2008, 20, 119-126.

(43) Campanini, M.; Trassin, M.; Ederer, C.; Erni, R.; Rossell, M. D. Buried In-Plane Ferroelectric Domains in Fe-Doped Single-Crystalline Aurivillius Thin Films. ACS Appl. Electron. Mater. 2019, 1, 10191028. 
(44) Boulle, A.; Guinebretìre, R.; Dauger, A. Highly localized strain fields due to planar defects in epitaxial $\mathrm{SrBi}_{2} \mathrm{Nb}_{2} \mathrm{O}_{9}$ thin films. J. Appl. Phys. 2005, 97, No. 073503.

(45) Zurbuchen, M. A.; Asayama, G.; Schlom, D. G.; Streiffer, S. K. Ferroelectric Domain Structure of $\mathrm{SrBi}_{2} \mathrm{Nb}_{2} \mathrm{O}_{9}$ Epitaxial Thin Films. Phys. Rev. Lett. 2002, 88, No. 107601.

(46) Zurbuchen, M. A.; Lettieri, J.; Fulk, S. J.; Jia, Y.; Carim, A. H.; Schlom, D. G.; et al. Bismuth volatility effects on the perfection of $\mathrm{SrBi}_{2} \mathrm{Nb}_{2} \mathrm{O}_{9}$ and $\mathrm{SrBi}_{2} \mathrm{Ta}_{2} \mathrm{O}_{9}$ films. Appl. Phys. Lett. 2003, 82, 4711.

(47) Moore, K.; Connell, E. N. O.; Keeney, L.; Downing, C.; Schmidt, M.; Nicolosi, V.; Bangert, U.; Conroy, M. Polar Vortexes and Charged Domain Walls in a Room Temperature Magnetoelectric Thin Film, 2020. DOI: 10.21203/rs.3.rs-114541/v3.

(48) Deepak, N.; Zhang, P. F.; Keeney, L.; Pemble, M. E.; Whatmore, R. W. Atomic vapor deposition of bismuth titanate thin films. J. Appl. Phys. 2013, 113, No. 187207.

(49) Seidel, J.; et al. Conduction at domain walls in oxide multiferroics. Nat. Mater. 2009, 8, 229-234.

(50) Meier, D.; Seidel, J.; Cano, A.; Delaney, K.; Kumagai, Y.; Mostovoy, M.; Spaldin, N. A.; Ramesh, R.; Fiebig, M. Anisotropic conductance at improper ferroelectric domain walls. Nat. Mater. 2012, $11,284-288$.

(51) Catalan, G.; Seidel, J.; Ramesh, R.; Scott, J. F. Domain wall nanoelectronics. Rev. Mod. Phys. 2012, 84, 119-156. 